\title{
HEPATOPROTECTIVE EFFECTS OF TRIBULUS TERRESTRIS, ASHWAGANDHA AND N-ACETYLCYSTEINE ON LIVER FIBROSIS IN CARBON TETRACHLORIDE-INDUCED RATS
}

\author{
DERYA ALTAY ${ }^{1 *}$, YASAR DOGAN $^{1}$, CEMAL ORHAN $^{2}$, MEHMET TUZCU $^{3}$, \\ NURHAN SAHIN ${ }^{2}$, IBRAHIM HANIFI OZERCAN ${ }^{4}$ and KAZIM SAHIN ${ }^{2}$
}

${ }^{1}$ Frrat University Faculty of Medicine, Department of Pediatric Gastroenterology, Hepatology and Nutrition, Turkey

${ }^{2}$ Firat University Faculty of Veterinary Medicine, Department of Animal Nutrition, Turkey

${ }^{3}$ Firat University Faculty of Science, Department of Biology, Turkey

${ }^{4}$ Firat University Faculty of Medicine, Department of Pathology, Turkey

\begin{abstract}
Fibrosis, which develops during the progression of liver damage, is the previous stage of cirrhosis. Carbon tetrachloride is one of the most commonly used hepatic toxins in experimental animal models of liver fibrosis. It was investigated the hepatoprotective effects of Tribulus terrestris, Ashwagandha and N-acetylcysteine in an experimental model of liver fibrosis induced by carbon tetrachloride in this study. Fifty Wistar rats were divided into five groups of 10 each, as follows: 1) Control, 2) Carbon tetrachloride, 3) Carbon tetrachloride plus N-acetylcysteine, 4) Carbon tetrachloride plus T. terrestris, and 5) Carbon tetrachloride plus Ashwagandha group. At the end of 6 weeks, the rats were sacrificed, and serum and tissue samples were collected. Aspartate aminotransferase, alanine aminotransferase, gamma-glutamyltranspeptidase, malondialdehyde, NF- $\kappa$ B, collagen 1 , nuclear factor erythroid-2-related factor 2 , tumor necrosis factor- $\alpha$ were analyzed, and histopathological evaluation was performed. There were no significant differences in serum aspartate aminotransferase and alanine aminotransferase levels of the $\mathrm{N}$-acetylcysteine-treated group versus those in the T. terrestris- and Ashwagandha-treated groups ( $\mathrm{p}>0.05$ ). Liver malondialdehyde levels were lower in the Nacetylcysteine-, T. terrestris- and Ashwagandha-treated groups than in the carbon tetrachloride-administered group $(\mathrm{p}<0.001)$. There were differences between groups in NF- $\kappa \mathrm{B}$, collagen 1 , nuclear factor erythroid-2related factor 2 and tumor necrosis factor- $\alpha$ levels $(\mathrm{p}<0.05$ ). In conclusion, T. terrestris, Ashwagandha and $\mathrm{N}-$ acetylcysteine had protective effects on the liver in this experimental fibrosis model. T. terrestris was a little more effective than Ashwagandha in combating liver fibrosis.
\end{abstract}

Keywords: Ashwagandha, carbon tetrachloride, N-acetylcysteine, Tribulus terrestris, liver fibrosis

Abbreviations: $\mathrm{CCl}_{4}$ : carbon tetrachloride, AST: aspartate aminotransferase, ALT: alanine aminotransferase, GGT: gamma-glutamyltranspeptidase, MDA: malondialdehyde, Nrf2: nuclear factor erythroid-2-related factor 2 TNF- $\alpha$ : tumor necrosis factor- $\alpha$, NAC: N-acetylcysteine

Fibrosis is a reversible consequence of chronic liver injury due to various etiologies. The development of fibrosis involves a complex wound-healing process, in which a large number of cell types triggered by cytokines, chemokines and non-peptide mediators interact (1). The progression of liver fibrosis results in cirrhosis, liver failure, and portal hypertension and often requires liver transplantation. The efficacy of drug treatment for liver fibrosis has been investigated in experimental models.

Chronic liver injury induced by carbon-tetrachloride $\left(\mathrm{CCl}_{4}\right)$ causes liver fibrosis in rats. $\mathrm{CCl}_{4}$ is one of the most commonly used hepatic toxins in experimental animal models of liver fibrosis and cirrhosis (2). The experimental rat model is biochemically and histologically similar to human liver cirrhosis. For this reason, the rat model of liver cirrhosis is very useful when investigating therapeutic efficacy of hepatoprotective drugs in humans.

Tribulus terrestris, also known as Gokshura, belongs to the Zygophyllaceae family and grows in subtropical regions. It is used in traditional medicine as a treatment for rheumatic diseases, hemorrhoids, renal and vesical stones, menorrhagia and impo-

\footnotetext{
* Corresponding author: e-mail: dr.deryaaltay@gmail.com
} 
tence. $T$. terrestris plant extract has potent diuretic and anti-hyperlipidemic effects. It contains steroids, saponins, flavonoids, alkaloids, unsaturated fatty acids, vitamins, tannins, aspartic acid, and glutamic acid. In addition, this plant extract has anti-bacterial, antioxidant and anti-toxic activities (3). Given the hypolipidemic and antioxidant effects of T. terrestris, it is thought to have hepatoprotective properties (4). Previous research demonstrated that tribulusamide A and B compounds were protective in hepatocytes in D-galactosamine-induced toxicity (5). T. terrestris, increase antioxidant enzymes such as superoxide dismutase, glutathione peroxidase-1, decrease malondialdehyde in serum, and reduces Caspase-3 expression which is a hydrolytic protease in a mouse model (6). In addition, T. terrestris provided protection against cytotoxicity in liver cancer cells by inhibiting oxidative stress (7).

Ashwagandha (Withania somnifera) belongs to the Solanaceae family of plants (8). Previous research reported that it had anti-inflammatory, antioxidative and anti-stress properties, which conferred hepatoprotection, in addition to immunomodulatory properties (8). Ashwagandha treatment altered levels of oxidative stress markers, significantly reducing lipid peroxidation and increasing superoxide dismutase and catalase activities, thereby removing free radicals (9-11).

$\mathrm{N}$-acetylcysteine, a precursor of L-cysteine and reduced glutathione, has been used as a mucolytic agent for many years. $\mathrm{N}$-acetylcysteine provides protection against acetaminophen-induced hepatotoxicity by replacing intracellular-reduced glutathione. $\mathrm{N}$-acetylcysteine easily penetrates the cell membrane. By enhancing cysteine intake, it increases the biosynthesis of reduced glutathione in cells, both in vivo and in vitro (12). Many studies have demonstrated hepatic injury-reducing effects of $\mathrm{N}$-acetylcysteine on $\mathrm{CCl}_{4}$-induced hepatotoxicity (12-15).

Interest in herbal medicines is increasing day by day. Two herbal extracts called $T$. terrestris and Ashwagandha commonly used in Chinese medicine were planned to compare with the hepatoprotective characteristic of a known medical drug N-acetylcysteine. The aim of the present study was to investigate the hepatoprotective effects of $T$. terrestris, Ashwagandha, and $\mathrm{N}$-acetylcysteine in an experimental rat model of liver fibrosis induced by $\mathrm{CCl}_{4}$. With this aim in mind, aspartate aminotransferase (AST), alanine aminotransferase (ALT), gammaglutamyltranspeptidase (GGT), malondialdehyde (MDA), NF- $\mathrm{KB}$, collagen 1, nuclear factor erythroid-2-related factor 2 (Nrf2), tumor necrosis fac- tor- $\alpha$ (TNF- $\alpha$ ), hepatic steatosis scores, necrosis, inflammation and levels of fibrosis were analyzed.

\section{MATERIALS AND METHODS}

\section{Animals}

Fifty 8 -week-old male Wistar rats weighing 180-220 gr were included in this study. The animals were provided by the Experimental Animal Centre of Fýrat University. The animals were housed in groups (pairs or triplets) in appropriate cages under a $12 \mathrm{~h}$ dark/12 h light cycle at an average temperature of $22^{\circ} \mathrm{C}$ in appropriate humidity conditions. All animals had access to pellet feed and water. Feeding of the animals ceased $12 \mathrm{~h}$ before the end of the experiment. All animals received human care and that study protocols comply with the institution's guidelines. The study was approved by the ethics committee of Frrat University (2016/93). This study was supported by Firat University Scientific Research Project Unit (TF.16.33).

\section{Experimental design}

The rats were randomly divided into the following five groups, with 10 animals in each group: group 1, control group; group 2, $\mathrm{CCl}_{4}$ treatment (fibrosis group); group $3, \mathrm{CCl}_{4}$ plus $\mathrm{N}$-acetylcysteine treatment; group $4, \mathrm{CCl}_{4}$ plus $T$. terrestris treatment; group 5, $\mathrm{CCl}_{4}$ plus Ashwagandha treatment. The animals in all five groups were fed a standard diet.

The rats were administered $\mathrm{CCl}_{4}(99.8 \%)$ (Merck $\mathrm{KGaA}$, Darmstadt, Germany). $\mathrm{CCl}_{4}$ was given with olive oil in a $1: 2$ ratio i.p. at a dose of $1.5 \mathrm{~mL} / \mathrm{kg}$ body weight twice weekly. N-acetylcysteine (Asist ampoule, $100 \mathrm{mg} / \mathrm{mL}$; Husnu Arsan, Istanbul, Turkey) was administered i.p. at a dose of $50 \mathrm{mg} / \mathrm{kg}$ body weight once daily. Dried fruit extract powder of $T$. Terrestris was extracted with a methanol-water mixture (70 : 30) (min: 40\% saponins), and dried roots of Ashwagandha were extracted using a methanol-water mixture (70:30) (min: $2.5 \%$ total withanolides). The purity of the extracts was $98 \%$. The extracts were dissolved in distilled water and administered via oral gavage once daily, with an optimal dose of $300 \mathrm{mg} / \mathrm{kg}$ body weight for T. terrestris and $500 \mathrm{mg} / \mathrm{kg}$ body weight for Ashwagandha for 6 weeks. The herbal extracts were supplied by Omni Active Health Technologies Pvt. Ltd. (Mumbai, India).

All animals were sacrificed under ether anesthesia at the end of 6 weeks. Blood and tissue samples were taken for analysis. The blood samples were centrifuged at $5.000 \mathrm{rpm}$ for $5 \mathrm{~min}$ to separate 
the serum and stored at $-20^{\circ} \mathrm{C}$ until the day of the study. The tissue samples were divided into two for molecular and histopathological studies. One-half of each sample was placed in $10 \%$ formalin, and the other half was frozen rapidly.

\section{Laboratory analysis}

Serum AST, ALT, and GGT levels were analyzed using a biochemical analyzer (Samsung LABGEO PT10, Samsung Electronics Co, Suwon, Korea). Reproducibility and device/method sensitivity of LABGEOPT10 were established according to IVR-PT06 guidelines. Serum and liver MDA levels were measured using the method described by Sahin et al. (16). Malondialdehyde (MDA) levels were measured using the fully automatic high-performance liquid chromatography (Shimadzu, Kyoto, Japan) equipped with a pump (LC-20 AD), an ultraviolet-visible detector (SPD-20A), an inertsil ODS3 C18 column $(250 \times 4.6 \mathrm{~mm}, 5 \mathrm{~m})$, a column oven (CTO-10ASVP), an autosampler (SIL-20A), a degasser unit (DGU-20A5) and a computer system with LC solution Software (Shimadzu) (16).

\section{Western blot analysis}

Protein (NF-kB, collagen 1, Nrf2, and TNF- $\alpha$ ) levels of liver tissues of the rats were analyzed by the Western blotting technique, as described by Sahin et al. (17). Liver tissues of the rats were removed after sacrification to analyze the protein expressions. Briefly, a $20 \%$ homogenate of the liver tissues were prepared in $1 \mathrm{~mL}$ ice-cold hypotonic buffer A containing 10 mM HEPES (pH 7.8), 10 $\mathrm{mM} \mathrm{KCl}, 2 \mathrm{mM}-\mathrm{MgCl}_{2}, 1 \mathrm{mM}$ dithiothreitol (DTT), $0.1 \mathrm{mM}$ EDTA and $0.1 \mathrm{mM}$ phenylmethylsulfonylfluoride. The homogenate was centrifuged at $5000 \mathrm{~g}$ and the resulting supernatant was collected, aliquoted in a small volume and to evaluate the expression of NF- $\kappa \mathrm{B}$, collagen 1, Nrf2 and TNF- $\alpha$ levels. All samples were analyzed in triplicates for each group. The samples were subjected to $10 \%$ SDS-PAGE and electrotransferred to a nitrocellulose membrane (Schleicher and Schuell Inc. Keene, NH, USA), which was soaked in methanol for $90 \mathrm{~min}$. The membranes were washed in PBS and blocked by a $1 \%$ bovine serum albumin (BSA) for $1 \mathrm{~h}$ prior to application of the primary antibody. Antibodies against NF- $\kappa \mathrm{B}$, collagen $1, \mathrm{Nrf} 2$, and $\mathrm{TNF}-\alpha$ (Abcam (Cambridge, UK)) were diluted (1: 1000) in the same buffer containing $0.05 \%$ Tween-20. Protein loading was controlled to sing a monoclonal mouse antibody against $\beta$-actin (A5316; Sigma). Bands were analyzed densitometrically using an image analyzing system (Image J: National Institute of Health, Bethesda, USA).

\section{Histopathological analysis}

Paraffin blocks were stained by hematoxylineosin and Masson's trichrome and were examined using an Olympus BX-50 microscope. The histopathological evaluation was performed by an experienced pathologist who was blinded to the study groups.

The percentage of steatotic cells was determined and classified as follows: less than $5 \%$, stage $0 ; 5-25 \%$, stage I; $25-50 \%$, stage II; $50-75 \%$, stage III; and more than $76 \%$, stage IV (18).

Inflammatory cells were randomly counted in at least 10 consecutive high-power fields $(\times 400$ magnification). The mean inflammatory cell number/ $/ \mathrm{mm}^{2}$ was obtained by dividing the total number by 10 (18).

Necrosis was quantified histologically by counting the number of inflammatory foci in at least 10 consecutive high-power fields. The mean necrotic field number $/ \mathrm{mm}^{2}$ was obtained by dividing the total number by 10 (18).

The degree of liver fibrosis was determined as follows, using a fibrosis scoring system (19): stage 0 , normal liver with no fibrosis; stage I, thick perivenular collagen and few collagen septa; stage II; thin septa, with incomplete bridges across the

Table 1. Comparison of ALT, AST and GGT levels of the groups.

\begin{tabular}{|c|c|c|c|c|c|}
\hline \multirow{2}{*}{} & \multicolumn{5}{|c|}{ Groups } \\
\cline { 2 - 6 } & Control & $\mathrm{CCl}_{4}$ & $\begin{array}{c}\mathrm{CCl}_{4}+ \\
\mathrm{N} \text {-acetylcysteine }\end{array}$ & $\begin{array}{c}\mathrm{CCl}_{4}+ \\
\text { Tribulus terrestris }\end{array}$ & $\begin{array}{c}\mathrm{CCl}_{4}+ \\
\text { Ashwagandha }\end{array}$ \\
\hline ALT, U/L & $70.14 \pm 3.63^{\mathrm{c}}$ & $301.71 \pm 43.61^{\mathrm{a}}$ & $185.67 \pm 23.95^{\mathrm{b}}$ & $177.40 \pm 14,47^{\mathrm{b}}$ & $258.20 \pm 36.14^{\mathrm{ab}}$ \\
\hline $\mathrm{AST}, \mathrm{U} / \mathrm{L}$ & $116.43 \pm 6.54^{\mathrm{c}}$ & $533.80 \pm 61.17^{\mathrm{a}}$ & $219.80 \pm 14.58^{\mathrm{bc}}$ & $290.60 \pm 47.73^{\mathrm{b}}$ & $348.20 \pm 18.68^{\mathrm{b}}$ \\
\hline GGT, U/L & $39.79 \pm 2.01^{\mathrm{e}}$ & $191.71 \pm 4.03^{\mathrm{a}}$ & $63.85 \pm 2.96^{\mathrm{d}}$ & $116.43 \pm 2.62^{\mathrm{c}}$ & $134.04 \pm 3.66^{\mathrm{b}}$ \\
\hline
\end{tabular}

ALT: Alanine aminotransferase; AST: Aspartate aminotransferase; GGT: Gamma-glutamyltranspeptidase. Data are means and standard error. Different superscripts $(a-c)$ indicate group mean differences $(p<0.05)$ 
portal regions; stage III; thin septa and extensive bridges; stage IV, thick septa with complete bridges across portal regions and a nodular appearance.

\section{Statistical analysis}

Data are expressed as mean \pm standard error. Differences among the groups were evaluated using an ANOVA, followed by Tukey's post hoc test. The alpha level of significance was set at $\mathrm{p}<0.05$.

\section{RESULTS}

Three rats in group 1, one rat in group 2, one rat in group 3, three rats in group 4 and four rats in group 5 died during the study, and the study was completed with 38 rats.

\section{Biochemical findings}

ALT, AST and GGT levels were measured as markers of liver function. As shown in Table1, serum ALT levels were significantly elevated in the Ashwagandha-treated group as compared with those in the control group $(\mathrm{p}=0.001)$. Serum ALT and AST levels in the $\mathrm{N}$-acetylcysteine-treated group were similar to those in the groups treated with $T$. terrestris and Ashwagandha, with no significant between-group difference ( $\mathrm{p}>0.05$ ). In contrast, GGT levels in the $\mathrm{N}$-acetylcysteine-treated group decreased as compared with those in the groups treated with $T$. terrestris and Ashwagandha ( $\mathrm{p}<$ 0.001).

As shown in Table 2, serum MDA levels were significantly higher in the $\mathrm{CCl}_{4}$-administered group as compared with those in the control group ( $\mathrm{p}<$ $0.001)$. There was a significant difference between groups in serum MDA levels $(\mathrm{p}<0.05)$. Liver MDA levels were lower in the N-acetylcysteine-, T. terrestris- and Ashwagandha-treated groups than in the $\mathrm{CCl}_{4}$-administered group $(\mathrm{p}<0.001)$.

\section{Western blot analysis findings}

In the molecular analysis, the NF- $\kappa \mathrm{B}$ level in the $\mathrm{N}$-acetylcysteine group was lower than that in the $\mathrm{CCl}_{4}$ group $(\mathrm{p}<0.001)$. However, there was no significant difference in $\mathrm{NF}-\kappa \mathrm{B}$ levels in the $\mathrm{N}$ acetylcysteine-, T. terrestris- and Ashwagandhatreated groups $(\mathrm{p}>0.05)$. The collagen 1 level in the $\mathrm{N}$-acetylcysteine group was lower than that in the $\mathrm{CCl}_{4}$ group ( $\left.\mathrm{p}=0.001\right)$, and it was lower in the T.terrestris-treated group than in the $\mathrm{CCl}_{4}$-administered group ( $\mathrm{p}=0.01)$. The Nrf2 level in the $\mathrm{N}$-acetylcysteine group was higher than that in the $\mathrm{CCl}_{4}$ group and Ashwagandha-treated group $(\mathrm{p}=0.005$ and $\mathrm{p}=$ 0.008 , respectively). However, there was no significant difference between groups in Nrf2 levels in the T. terrestris- and Ashwagandha-treated groups ( $\mathrm{p}>$

Table 2. Comparison of serum and liver MDA levels of the groups.

\begin{tabular}{|c|c|c|c|c|c|}
\hline & \multicolumn{5}{|c|}{ Groups } \\
\hline & Control & $\mathrm{CCl}_{4}$ & $\begin{array}{c}\mathrm{CCl}_{4}+ \\
\mathrm{N} \text {-acetylcysteine }\end{array}$ & $\begin{array}{c}\mathrm{CCl}_{4}+ \\
\text { Tribulus terrestris }\end{array}$ & $\begin{array}{c}\mathrm{CCl}_{4}+ \\
\text { Ashwagandha }\end{array}$ \\
\hline Serum MDA, $\mu \mathrm{mol} / \mathrm{mL}$ & $0.95 \pm 0.04^{\mathrm{e}}$ & $2.93 \pm 0.04^{\mathrm{a}}$ & $1.30 \pm 0.02^{\mathrm{d}}$ & $1.53 \pm 0,04^{\mathrm{c}}$ & $1.92 \pm 0.02^{\mathrm{b}}$ \\
\hline Liver MDA, nmol/g & $2.54 \pm 0.10^{\mathrm{d}}$ & $6.17 \pm 0.37^{\mathrm{a}}$ & $3.54 \pm 0.09^{c}$ & $4.02 \pm 0.07 b^{c}$ & $4.42 \pm 0.06^{\mathrm{b}}$ \\
\hline
\end{tabular}

MDA: Malondialdehyde. Data are means and standard error. Different superscripts (a-d) indicate group mean differences $(\mathrm{p}<0.05)$

Table 3. Comparison of histopathological analysis of the groups.

\begin{tabular}{|c|c|c|c|c|c|}
\hline \multirow{2}{*}{} & \multicolumn{5}{|c|}{$\mathrm{Groups}^{*}$} \\
\cline { 2 - 6 } & Control & $\mathrm{CCl}_{4}$ & $\begin{array}{c}\mathrm{CCl}_{4}+ \\
\text { N-acetylcysteine }\end{array}$ & $\begin{array}{c}\mathrm{CCl}_{4}+ \\
\text { Tribulus terrestris }\end{array}$ & $\begin{array}{c}\mathrm{CCl}_{4}+ \\
\text { Ashwagandha }\end{array}$ \\
\hline Steatosis & $0.00 \pm 0.00^{\mathrm{c}}$ & $1.30 \pm 0.15^{\mathrm{ab}}$ & $1.00 \pm 0.27^{\mathrm{ab}}$ & $0.67 \pm 0.21^{\mathrm{bc}}$ & $1.63 \pm 0.18^{\mathrm{a}}$ \\
\hline Fibrosis & $0.00 \pm 0.00^{\mathrm{c}}$ & $3.30 \pm 0.15^{\mathrm{a}}$ & $1.63 \pm 0.26^{\mathrm{b}}$ & $2.50 \pm 0.34^{\mathrm{ab}}$ & $2.75 \pm 0.16^{\mathrm{a}}$ \\
\hline Necrosis $\left(\mathrm{mm}^{2}\right)$ & $0.40 \pm 0.24^{\mathrm{b}}$ & $2.70 \pm 0.21^{\mathrm{a}}$ & $0.50 \pm 0.19^{\mathrm{b}}$ & $0.67 \pm 0.21^{\mathrm{b}}$ & $1.75 \pm 0.25^{\mathrm{a}}$ \\
\hline Inflammation $\left(\mathrm{mm}^{2}\right)$ & $2.00 \pm 0.89^{\mathrm{b}}$ & $53.50 \pm 5.78^{\mathrm{a}}$ & $16.00 \pm 2.79^{\mathrm{b}}$ & $15.00 \pm 3.65^{\mathrm{b}}$ & $36.25 \pm 4.79^{\mathrm{a}}$ \\
\hline
\end{tabular}

Data are means and standard error. Different superscripts $(\mathrm{a}-\mathrm{d})$ indicate group mean differences $(\mathrm{p}<0.05)$ 

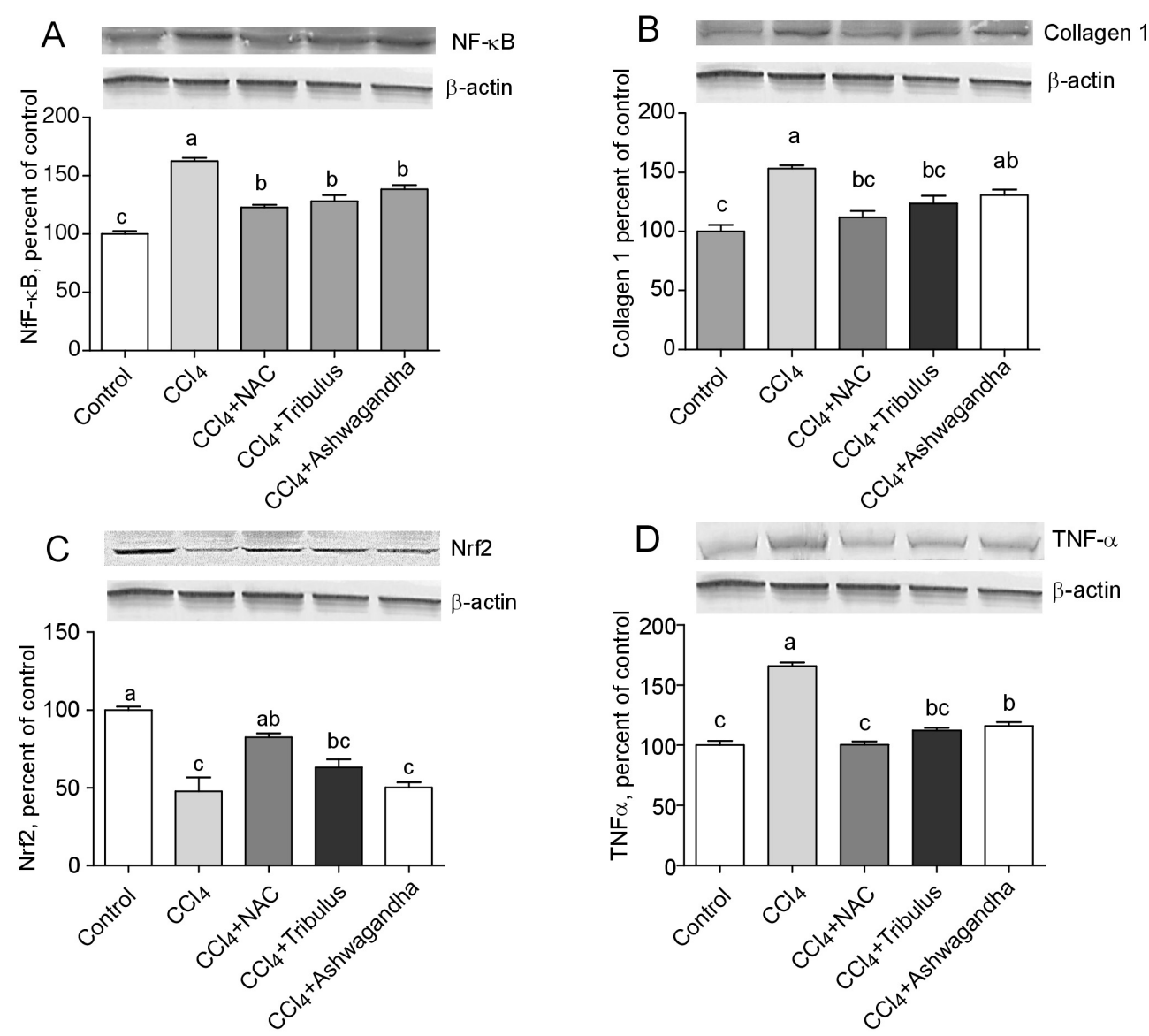

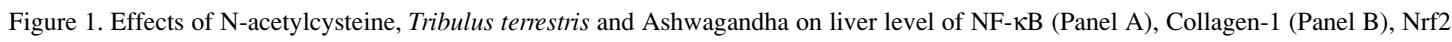
(Panel C), and TNF $\alpha$ (Panel D) in $\mathrm{CCl}_{4}$-induced rats. The intensity of the bands was quantified by densitometric analysis and $\beta$-Actin was included to ensure equal protein loading. Data are expressed as a ratio of normal control value (set to 100\%). Each bar represents the mean and standard error. Blots were repeated at least 3 times $(n=3)$. Different superscripts on top of each bar $(a, b$, c) indicate group mean differences $(p<0.05)$

$0.05)$. There was a significant difference in TNF- $\alpha$ levels in the $\mathrm{N}$-acetylcysteine- and Ashwagandhatreated groups $(\mathrm{p}=0.02)$ (Fig. 1).

\section{Histopathological findings}

The levels of steatosis, fibrosis, necrosis, and inflammation in the groups are shown in Table 3. As expected, hepatic injury markedly increased in the $\mathrm{CCl}_{4}$-administered group as compared with the level of injury in the other groups. The steatosis level in the Ashwagandha-treated group was higher than that in the T. terrestris-treated group. However, fibrosis was similar in both the T. Terrestris and Ashwagandha groups, with no significant between-group difference $(p>0.05)$. Necrosis and inflammation were elevated in the Ashwagandha-treated group as compared with these parameters in the T. terrestristreated group $(\mathrm{p}<0.05)$. Picture 1 shows the results of hematoxylin-eosin staining in the various groups.

\section{DISCUSSION}

This study investigated the effects of $T$. terrestris and Ashwagandha extracts on liver fibrosis induced by $\mathrm{CCl}_{4}$, a well-known hepatotoxic industrial solvent, and herbal extracts were administered to reverse fibrosis. The results were then compared with those obtained by $\mathrm{N}$-acetylcysteine treatment.

Although previous studies examined the hepatoprotective effects of T. terrestris (20-22), there have been no studies of $T$. terrestris activity in a rat model of $\mathrm{CCl}_{4}$-induced fibrosis. Based on the find- 
ings of these previous studies, the optimal dose of T. terrestris in the present study was considered 300 $\mathrm{mg} / \mathrm{kg}$ body weight. The $\mathrm{N}$-acetylcysteine and Ashwagandha doses administered to rats in the present study of $\mathrm{CCl}_{4}$-induced fibrosis were based on the findings of previous studies of the hepatoprotective dose of $\mathrm{N}$-acetylcysteine $(23,24)$. N-acetylcysteine exerts a detoxification effect by stimulating glutathione synthesis. It has direct radical-scavenging properties and combats acetaminophen toxicity. It is used clinically as a hepatoprotective drug and has mucolytic properties (25). And also, N-acetylcysteine inhibits activation of $\mathrm{NF}-\kappa \mathrm{B}$, responsible for the inflammatory process (26). In the present study, $\mathrm{N}$-acetylcysteine was used as a standard hepatoprotective agent, and we compared its properties with those of other herbal extracts.
Ansari et al. (27) reported that $T$. terrestris was hepatoprotective in acetaminophen-induced rats. They administered T. terrestris at a dose of 500 $\mathrm{mg} / \mathrm{kg}$ for 6 days and showed a decrease in liver enzymes. In an experimental fatty liver model induced by a high fructose diet, Almasi et al. (4) reported that administered at doses of 500, 700 and $1000 \mathrm{mg} / \mathrm{kg}$ for 5 weeks led to a decrease in liver enzymes and hepatosteatosis in accordance with an increase in the dose. Their study was published after our study was completed. Kavitha et al. (22) reported that T. terrestris $(250 \mathrm{mg} / \mathrm{kg})$ was hepatoprotective and antioxidative against acetaminopheninduced toxicity. In the present study, we administered T. terrestris at a dose of $300 \mathrm{mg} / \mathrm{kg}$ for 6 weeks. At the end of this study, it was found that decreased of AST and ALT levels of the groups given herbal

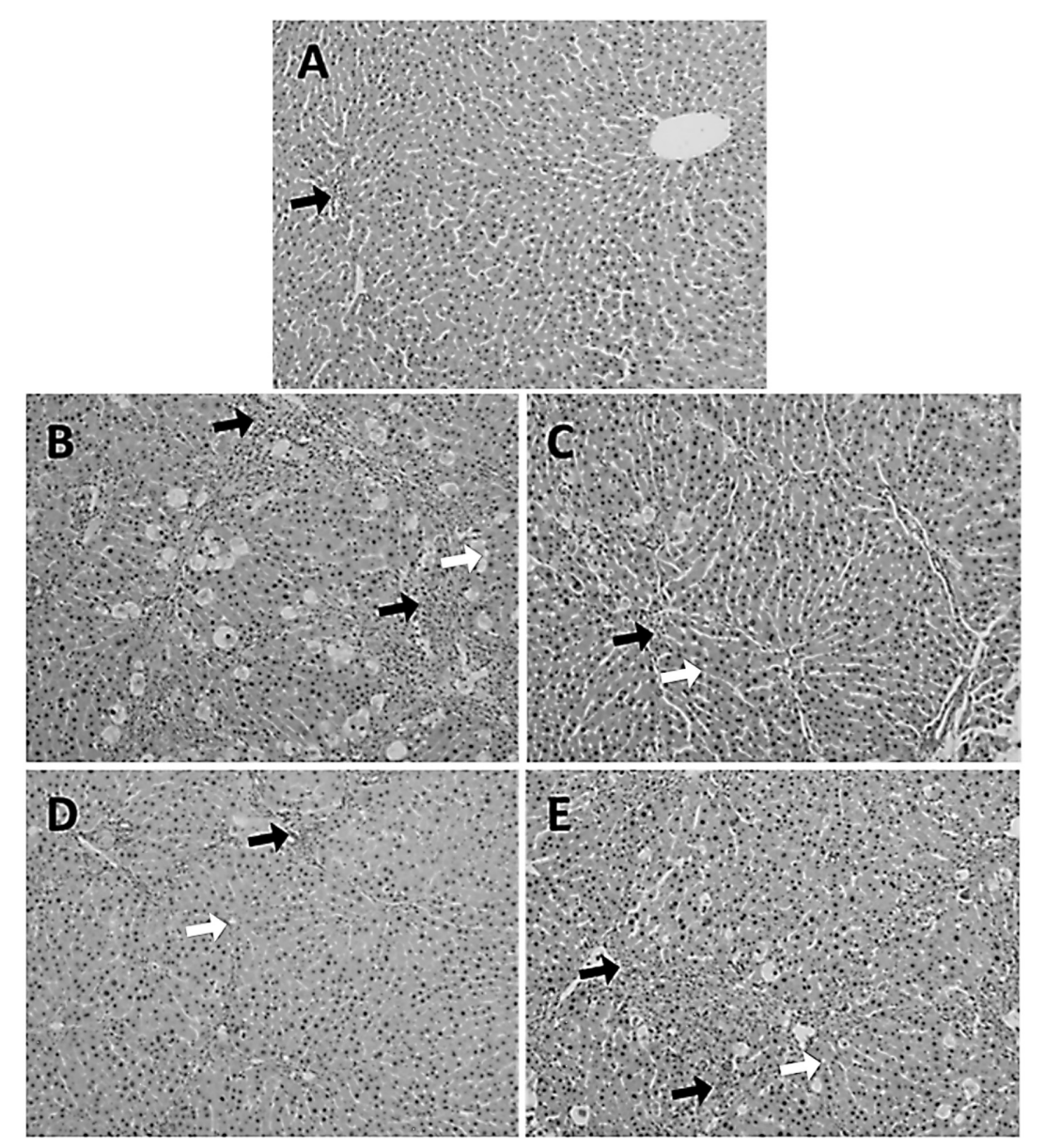

Picture 1. Evaluation of liver tissue samples after hematoxylin-eosin staining $(\times 100)$ in groups. A: Rare inflammatory cells in the normal portal area (arrow) in the control group, B: Dense inflammatory cells (black arrow) and steatosis (white arrow) in the $\mathrm{CCl}_{4}$ administered group, C: Mild inflammatory cell infiltration (black arrow) and steatosis (white arrow) in the $\mathrm{CCl}_{4}$ plus $\mathrm{N}$-acetylcysteine administered group, D: Mild inflammatory cell infiltration (black arrow) and steatosis (white arrow) in the $\mathrm{CCl}_{4}$ plus Tribulus terrestris administered group, E: Dense inflammatory cells (black arrow) and steatosis (white arrow) in the $\mathrm{CCl}_{4}$ plus Ashwagandha administered group 
extracts were similar with $\mathrm{N}$-acetylcysteine administered group, but GGT levels were the lowest in Nacetylcysteine administered group compared to the other two herbal extracts. The rate of hepatosteatosis was also low in the T. Terrestris administered group compared to the other two chemicals.

Sultana et al. (8) administered Ashwagandha at a dose of $500 \mathrm{mg} / \mathrm{kg}$ for 22 days to rats in which gentamicin toxicity had been induced and showed a significant decrease in liver enzymes. Another study reported that as compared with silymarin treatment in paracetamol-induced hepatotoxicity, Ashwagandha treatment administered in a single dose of 500 and $1000 \mathrm{mg} / \mathrm{kg}$ resulted in an improvement in liver enzymes (9). Mansour et al. (28) showed that Ashwagandha treatment at a dose of $100 \mathrm{mg} / \mathrm{kg}$ for 7 days led to a decrease in liver enzymes and DNA damage in liver induced by gamma irradiation and attributed this decrease to the antioxidant properties of Ashwagandha. The same study reported that Ashwagandha induced hem oxygenase activity, an essential step in cellular adaptation in stress. Elberry et al. (24) reported that $500 \mathrm{mg} / \mathrm{kg}$ of Ashwagandha treatment in $\mathrm{CCl}_{4}$-induced rats for 4 weeks had protective effects in the liver, as shown by biochemical, antioxidant and histopathological findings (24). In the present study, we administered Ashwagandha at a dose of $500 \mathrm{mg} / \mathrm{kg}$ and T. Terrestris at a dose of 300 $\mathrm{mg} / \mathrm{kg}$ for 6 weeks and compared the effects with those of N-acetylcysteine treatment. AST and ALT levels in the group treated with Ashwagandha were similar to those in the groups treated with $T$. terrestris and $\mathrm{N}$-acetylcysteine.

In $\mathrm{CCl}_{4}$-induced intoxication, antioxidants and anti-inflammatory agents provide detoxification by clearing free radicals via the release of active oxygen and neutralizing lipid peroxides (29). MDA, a lipid peroxidation product, is a biomarker of oxidative stress (30). Elberry et al. (24) showed that Ashwagandha caused a decrease in MDA levels and that this was due to its antioxidant effect. In our study, serum and tissue MDA levels decreased in both groups given herbal extracts, with the decrease more apparent in the group treated with $T$. terrestris. $\mathrm{NF}-\kappa \mathrm{B}$ plays an important role in the regulation of the inflammatory pathway in the liver (31). Withanolides in the Ashwagandha extract inhibit cyclooxygenase enzymes, lipid peroxidation and proliferation of tumor cells, thereby inactivating the $\mathrm{NF}-\kappa \mathrm{B}$ pathway and reducing the inflammatory process. NF- $\kappa \mathrm{B}$ regulates TNF- $\alpha$ expression, which plays a critical role in inflammation. The level of pro-inflammatory cytokines, such as TNF- $\alpha$, is quite high in drug-induced hepatotoxicity (32).
Devkar et al. (33) reported that Ashwagandha treatment alleviated inflammation by inhibiting TNF- $\alpha$. Elberry et al. (24) stated that steroidal lactones and withanolides in Ashwagandha conferred an antioxidant effect. In the current study, NF- $\kappa \mathrm{B}$ levels were lower in $\mathrm{N}$-acetylcysteine-treated group as compared with that in the $\mathrm{CCl}_{4}$-administered group. However, NF- $\kappa \mathrm{B}$ levels in the groups treated with N-acetylcysteine, T. terrestris and Ashwagandha were not significantly different. Based on these findings, it can be concluded that $T$. terrestris and Ashwagandha, as well as $\mathrm{N}$-acetylcysteine, can inactivate NF- $\kappa \mathrm{B}$. In addition, although TNF- $\alpha$ levels in the control and $\mathrm{N}$-acetylcysteine-treated groups were similar, they were higher in the group given Ashwagandha. In contrast, the levels of Nrf2, a major regulator of the antioxidant response, produce anti-inflammatory changes and activates antioxidant genes. And also, Nrf2 protects cells from stressors, including endogenous substances, reactive oxygen species, radiation, and environmental toxins (34). Palliyaguru et al. (35) reported that Nrf2 was played an important role in cytoprotection and hepatoprotection of Ashwagandha via a Keap1-independent and Pten/Pi3k/Akt-dependent mechanisms. In the current study, Nrf2 was not increased in the group treated with Ashwagandha as well as T. terrestris. T. terrestris was a bit more effective than Ashwagandha in activating the Nrf2 pathway. It was thought that the stimulation of the Nrf2 pathway may contribute to the antioxidant activity of the $T$. terrestris.

A healthy liver contains low levels of collagen type I, III and IV. In a fibrotic liver, the number of collagen increases, with advanced liver fibrosis characterized by an increase in type I collagen (36). In the present study, the similarity in collagen $1 \mathrm{lev}$ els in the $\mathrm{N}$-acetylcysteine-, T. terrestris-, and Ashwagandha-treated groups suggests that both $T$. terrestris and Ashwagandha have fibrosis-reducing effects.

In the present study, in the histopathological evaluation, the most prominent fibrosis, steatosis, and inflammation were observed in the $\mathrm{CCl}_{4}$-administered group, as expected. Fibrosis levels in the $T$. terrestris- and Ashwagandha-treated groups were similar, but inflammation was less severe in the Ashwagandha-treated group. Previous research demonstrated that $\mathrm{N}$-acetylcysteine provided protection against hepatic fibrosis in cirrhotic rat liver (13). Studies also showed that T. terrestris (4) and Ashwagandha (9) were effective in combating fibrosis in models of hepatotoxicity.

A limitation of the present study was that we did not compare the activities of herbal extracts at 
different doses. The activity of $T$. terrestris was determined only at a dose of $300 \mathrm{mg} / \mathrm{kg}$ body weight, whereas that of Ashwagandha was determined only at a dose of $500 \mathrm{mg} / \mathrm{kg}$ body weight.

In conclusion, this is the first study to demonstrate the effects of $T$. terrestris in experimental liver fibrosis induced by $\mathrm{CCl}_{4}$. We conclude that $T$. terrestris and Ashwagandha may have potential as hepatoprotective agents due to their positive effects in reversing liver fibrosis, with $T$. terrestris having slightly better hepatoprotective properties than Ashwagandha. More experimental and clinical studies are needed to shed light on the potential efficacy of T. terrestris and Ashwagandha as hepatoprotective agents.

\section{Conflict of interest}

The authors declare no conflicts of interest.

\section{REFERENCES}

1. Gressner A.M., Weiskirchen R.: J. Cell. Mol. Med. 10, 76 (2006).

2. Lee G.P., Jeong W.I., Jeong D.H., Do S.H., Kim T.H., Jeong K.S.: Anticancer Res. 25, 1029 (2005).

3. Akram M., Asif H.M., Akhtar N., Shah P.A., Uzair M. et al.: J. Med. Plant. Res. 5, 3601 (2011).

4. Almasi F., Khazaei M., Chehrei S., Ghanbari A.: Int. J. Morphol. 35, 345 (2017).

5. Asmari A.K., Elaiwi A.M., Athar T., Tariq M., Eid A., Asmary S.M.: Evid. Based Complement. Alternat. Med. 2014, 1 (2014).

6. Zhu W., Du Y., Meng H., Dong Y., Li L.: Chem. Cent. J. 11, 60 (2017).

7. Chhatre S., Nesari T., Somani G., Kanchan D., Sathaye S.: Pharmacogn. Rev. 8, 45 (2014).

8. Sultana N., Shimmi S.C., Parash T.H., Akhtar J.: J. Bangladesh Soc. Physiol. 7, 1 (2012).

9. Sabina E.P., Rasool M., Vedi M., Navaneethan D., Ravichander M. et al.: Int. J. Pharm. Pharm. Sci. 5, 648 (2013).

10. Dhuley J.N.: J. Ethnopharmacol. 60, 173 (1998).

11. Panda S., Kar A.: Indian J. Physiol. Pharmacol. 41, 424 (1997).

12. Galicia-Moreno M., Rodríguez-Rivera A., Reyes-Gordillo K., Segovia J., Shibayama M. et al.: Eur. J. Gastroenterol. Hepatol. 21, 908 (2009).

13. Pereira-Filho G., Ferreira C., Schwengber A., Marroni C., Zettler C., Marroni N.: Arq. Gastroenterol. 45, 156 (2008).
14. Maksimchik Y.Z., Lapshina E.A., Sudnikovich E.Y., Zabrodskaya S.V., Zavodnik I.B.: Cell. Biochem. Funct. 26, 11 (2008).

15. Vercelino R., Crespo I., de Souza G.F., Cuevas M.J., de Oliveira M.G. et al.: J. Mol. Med. 88, 401 (2010).

16. Sahin K., Orhan C., Akdemir F., Tuzcu M., Gencoglu H. et al.: BMC Complement. Altern. Med. 16, 1 (2016).

17. Sahin K., Orhan C., Akdemir F., Tuzcu M., Sahin N. et al.: Food Chem. Toxicol. 107, 270 (2017).

18. Kuzu N., Metin K., Daglı A.F., Akdemir F., Orhan C. et al.: Mediat. Inflamm. 2007, 6 pages (2007).

19. Rivera C.A., Bradford B.U., Hunt K.J., Adachi Y., Schrum L.W. et al.: Am. J. Physiol. Gastrointest. Liver Physiol. 281, 200 (2001).

20. Kumar P., Singh P.: Indian J. Biochem. Biophys. 53, 179 (2016).

21. Harraz F.M., Ghazy N.M., Hammoda H.M., Nafeaa A.A., Abdallah I.I.: J. Phys. Pharm. Adv. 5, 787 (2015).

22. Kavitha P., Ramesh R., Bupesh G., Stalin A., Subramanian P.: In Vitro Cell. Dev. Biol. Anim. 47, 698 (2011).

23. Demiroren K., Dogan Y., Kocamaz H., Ozercan I.H., Ilhan S. et al.: Clin. Res. Hepatol. Gastroenterol. 38, 63 (2014).

24. Elberry A.A., Harraz F.M., Ghareib S.A., Nagy A.A., Gabr S.A., Suliaman M.I.: J. Basic Clin. Pharm. 1, 247 (2010).

25. Kelly G.S.: Alt. Med. Rev. 3, 114 (1998).

26. Zafarullah M., Li WQ., Sylvester J., Ahmad M.: Cell. Mol. Life Sci. 60, 6 (2003).

27. Ansari J.A., Sayyed A.M., Balamurgan R.M.: Euroasian J. Hepatogastroenterol. 3, 15 (2013).

28. Mansour H.H., Hafez H.F.: Ecotoxicol. Environ. Saf. 80, 14 (2012).

29. Adewale O.B., Adekeye A.O., Akintayo C.O., Onikanni A., Saheed S.: J. Phytopharmacol. 3, 118 (2014).

30. Singh Z., Karthigesu I.P., Singh P., Kaur R.: Iran. J. Publ. Health 43, 7 (2014).

31. Luedde T., Schwabe R.F.: Nat. Rev. Gastroenterol. Hepatol. 8, 108 (2011).

32. Shimmi S.C., Jahan N., Sultana N.: J. Bangladesh Soc. Physiol. 6, 84 (2011).

33. Devkar S.T., Kandhare A.D., Zanwar A.A., Jagtap S.D., Katyare S.S. et al.: Pharm. Biol. 54, 2394 (2016).

34. Huang Y., Li W.,Su Z.Y., Kong A.N.: J. Nutr. Biochem. 26, 1401 (2015). 
35. Palliyaguru D.L., Chartoumpekis D.V., Wakabayashi N., Skoko J.J., Yagishita Y.: Free Radic. Biol. Med. 101, 116 (2016).
36. Pierce R.A., Glaug M.R., Greco R.S., Mackenzie J.W., Boyd C.D., Deak S.B.: J. Biol. Chem. 262, 1652 (1987).

Received: 7.12.2018 